\title{
The H-alpha luminosity function at redshift $2.2^{\star}$ A new determination using VLT/HAWK-I
}

\author{
M. Hayes ${ }^{1}$, D. Schaerer ${ }^{1,2}$, and G. Östlin ${ }^{3}$ \\ 1 Geneva Observatory, University of Geneva, 51 chemin des Maillettes, 1290 Sauverny, Switzerland \\ e-mail: matthew.hayes@unige.ch \\ ${ }^{2}$ Laboratoire d'Astrophysique de Toulouse-Tarbes, Université de Toulouse, CNRS, 14 Avenue E. Belin, 31400 Toulouse, France \\ 3 Oskar Klein Center for Cosmoparticle physics, Department of Astronomy, Stockholm University, 10691 Stockholm, Sweden
}

Received 31 August 2009 / Accepted 7 December 2009

\begin{abstract}
We aim to place new, strengthened constraints on the luminosity function $(L F)$ of $\mathrm{H}$-alpha $(\mathrm{H} \alpha)$ emitting galaxies at redshift $z \approx 2.2$, and to further constrain the instantaneous star-formation rate density of the universe $\left(\dot{\rho}_{\star}\right)$. We have used the new $H A W K-I$ instrument at $E S O-V L T$ to obtain extremely deep narrow-band (line; NB2090) and broad-band (continuum; $K_{\mathrm{s}}$ ) imaging observations. The target field is in the GOODS-South, providing us with a rich multi-wavelength auxiliary data set, which we utilise for redshift confirmation and to estimate dust content. We use this new data to measure the faint-end slope $(\alpha)$ of $L F(\mathrm{H} \alpha)$ with unprecedented precision. The data are well fit by a Schechter function and also a single power-law, yielding $\alpha=(-1.72 \pm 0.20)$ and $(-1.77 \pm 0.21)$, respectively. Thus we are able to confirm the steepening of $\alpha$ from low- to high- $z$ predicted by a number of authors and observed at other wavelengths. We combine our $L F$ data-points with those from a much shallower but wider survey at $z \sim 2.2$ (Geach et al. 2008), constructing a $L F$ spanning a factor of 50 in luminosity. Re-fitting the Schechter parameters, we obtain $\log L_{\star}=(43.07 \pm 0.22) \mathrm{erg} \mathrm{s}^{-1} ; \log \phi_{\star}=$ $(-3.45 \pm 0.52) \mathrm{Mpc}^{-3} ; \alpha=(-1.60 \pm 0.15)$. We integrate over $L F(\mathrm{H} \alpha)$ and apply a correction for dust attenuation to determine the instantaneous cosmic star-formation rate density at $z \sim 2$ without assuming $\alpha$ or extrapolating it from lower- $z$. Our measurement of $\dot{\rho}_{\star}$ is $(0.215 \pm 0.090) M_{\odot} \mathrm{yr}^{-1} \mathrm{Mpc}^{-3}$, integrated over a range of $37 \leq \log \left(L_{\mathrm{H} \alpha} / \mathrm{erg} \mathrm{s}^{-1}\right) \leq 47$.
\end{abstract}

Key words. galaxies: fundamental parameters - galaxies: high-redshift - galaxies: evolution - galaxies: starburst galaxies: luminosity function, mass function

\section{Introduction}

Since the evolution in the rate of cosmic star-formation was first plotted (Lilly et al. 1996; Madau et al. 1996), the literature has been awash with studies adding further points to the diagram. To estimate this quantity, one typically needs to find a number of galaxies by a given method and estimate their density in both space and luminosity (i.e. the luminosity function, $L F$ ). Integration of $L F \cdot L \cdot \mathrm{d} L$ thus provides the volume-averaged luminosity density $\left(\rho_{L}\right)$ and, if $L$ is a suitably calibrated measure of star-formation rate (SFR), $\rho_{L}$ converts directly into the volumetric rate of star-formation $\left(\dot{\rho}_{\star}\right)$.

A favourite tracer among low- $z$ observers for estimating starformation is the $\mathrm{H} \alpha$ emission line due to its simple physics, high intrinsic brightness, and convenient rest-wavelength at $6563 \AA$. At $z \gtrsim 2.5$, however, the $\mathrm{H} \alpha$ line shifts out of the $K$-band, making it highly inefficient for galaxy evolution studies. Fortunately at this $z$, selection by either the Ly $\alpha$ line or continuum $(B M / B X$, $B z K)$ criteria becomes possible, but unfortunately a different population of galaxies may be recovered, introducing biases in selected star-formation rate/history, dust content, etc. More specifically with regard to SFR, continuum luminosities come to equilibrium (and are therefore calibrated) over very different time-scales to nebular lines: $\gtrsim 100$ and $\sim 10$ Myr respectively, implying that lines are more sensitive to the instantaneous SFR

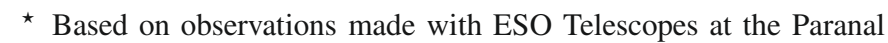
Observatory under programme ID 081.A-0932. (e.g. Kennicutt 1998). Ly $\alpha$ enables surveys to go much further in redshift and has identical production physics, but is a resonance line and undergoes a complex radiation transport, which renders it an unreliable tracer of intrinsic properties. From a purely physical perspective, $\mathrm{H} \alpha$ is a far preferable tool.

Luminosity functions are typically parameterised and compared using the Schechter function ${ }^{1}$ in which the luminosity distribution below (above) the characteristic luminosity, $L_{\star}$, is dominated by a power-law (exponential). When fitting, the three Schechter parameters are degenerate, and strong constraints are only obtained by sampling above and significantly below $L_{\star}$. Many attempts have been made to pin down $L F(\mathrm{H} \alpha)$ and its evolution with redshift from $z \approx 0$ (e.g. Gallego et al. 1995), through intermediate- $z$ (Yan et al. 1999; Hopkins et al. 2000; Tresse et al. 2002; Sobral et al. 2009; Shim et al. 2009), with the first $z=2$ limits placed by Bunker et al. (1995). It is only in recent years that $z=2$ surveys have been fruitful, with the most significant study being that of Geach et al. (2008, hereafter G08). However, this study was a wide, shallow survey designed to find the bright objects and determine $L_{\star}$, but does not permit an estimate of the faint-end slope.

With the wide-field $(7.5 \times 7.5) H A W K-I$ instrument (Pirard et al. 2004; Casali et al. 2006) at ESO-VLT we have obtained the deepest narrowband $\mathrm{H} \alpha$ observations to date as part of a programme to study $\mathrm{H} \alpha$ and $\mathrm{Ly} \alpha$ emitting galaxies from a single

$\overline{{ }^{1} \Phi(L) \cdot \mathrm{d} L=\phi_{\star} \cdot\left(L / L_{\star}\right)^{\alpha}} \cdot \exp \left(L / L_{\star}\right) \cdot \mathrm{d} L / L_{\star}$. 
volume at $z \sim 2.2$ (Hayes et al., submitted). These $\mathrm{H} \alpha$ data alone enable us to study the faint-end of $L F(\mathrm{H} \alpha)$ at unprecedented depths, tightening constraints on the overall $L F(\mathrm{H} \alpha)$, and providing the content of this Letter. In Sect. 2 we briefly describe the data, reduction, and selection; in Sect. 3 we present the $z \sim 2 \mathrm{H} \alpha$ luminosity functions we derive and the constraints on $\dot{\rho}_{\star}$; and in Sect. 4 we briefly summarise. Throughout we adopt a cosmology of $\left(H_{0}, \Omega_{\mathrm{M}}, \Omega_{\Lambda}\right)=\left(70 \mathrm{~km} \mathrm{~s}^{-1} \mathrm{Mpc}^{-1}, 0.3,0.7\right)$, the $\operatorname{SFR}(\mathrm{H} \alpha)$ calibration of Kennicutt (1998), derived for a Salpeter initial mass function in the mass range $\left(M_{\mathrm{lo}}, M_{\mathrm{hi}}\right)=$ $(0.1,100) M_{\odot}$ and solar abundances, and the $A B$ magnitude system (Oke \& Gunn 1983).

\section{The data}

\subsection{Observations and reduction}

A field in the GOODS South (Giavalisco et al. 2004) was selected to maximise the depth and quality of the auxiliary data. The central pointing was $\alpha=03: 32: 32.88 ; \delta=-27: 47: 16$; $\mathrm{PA}=-44 \mathrm{deg}$. The field was observed in service mode between 08 September and 21 August 2008 on a single dithered pointing. The $N B 2090$ filter $\left(\lambda_{\mathrm{c}}=2.095 \mu \mathrm{m} ; \Delta \lambda=0.019 \mu \mathrm{m}\right)$ was used to isolate emission line candidates, using a total integration time of $60000 \mathrm{~s}$. For the continuum we obtained $7500 \mathrm{~s}$ in $H A W K-I K_{\mathrm{s}}$ band, which we combined with the publicly available ISAAC $K_{\mathrm{s}}$ data from the GOODS/ESO Imaging Survey.

Full details of the reduction process are beyond the scope of this Letter and will be presented in a forthcoming article (Hayes et al. 2010). For brevity, the EsoRex HAWK-I pipeline was used on the individual frames for bias-subtraction, flat-fielding, and subtraction of the sky with temporally adjacent image pairs. Custom scripts were then used to mask cross-talk artifacts, register and co-add the individual frames. We modelled the point spread function (PSF) in the final image and determined a seeing of $0.89^{\prime \prime}$. To estimate the limiting magnitude we added artificial point-sources (full width at half maximum set to the measured seeing) to the images with the addstar task in noao/iraf and tested their recovery using SExtractor (Bertin \& Arnouts 1996; see Sect. 2.2). It should be noted that the limiting magnitude for extended objects will be somewhat shallower, but also that at $z=2.2$, one seeing disc corresponds to a physical scale of $7.4 \mathrm{kpc}$. Using SExtractor mag_auto magnitudes we determined a $5 \sigma$ limiting magnitude of 24.6 in NB2090. By computing the product of this flux density and $\Delta \lambda_{N B 2090}$, this corresponds to a line flux of $6.85 \times 10^{-18} \mathrm{erg} \mathrm{s}^{-1}$, if all the NB2090 flux comes from $\mathrm{H} \alpha$ and falls at the peak of the filter throughput. This assumption, valid for perfect top-hat bandpasses, holds well for our bandpass, which has steep edges by narrowband standards: the full width at $80 \%$ transmission is over $80 \%$ the $F W H M$. This limiting flux corresponds to $S F R=1.9 M_{\odot} \mathrm{yr}^{-1}$. The $K_{\mathrm{s}} 5 \sigma$ limit is 25.4 .

\subsection{Selection of $H$-alpha emitting candidate galaxies}

Source detection was performed in the on-line image using SExtractor, where we required a minimum of 12 contiguous pixels ( plate-scale $=0$. . $^{\prime} 106 \mathrm{px}^{-1}$ ) to reside above the background noise by a signal-to-noise $(S / N=3)$. Aperture-matched $K_{\mathrm{s}}$ photometry was performed using "double image" mode. We selected emission line candidates based upon two criteria, the first of which was a minimum rest-frame equivalent width, $E W_{\mathrm{H} \alpha}=$ $20 \AA$. Furthermore, in order to prevent over-contamination from $K_{\mathrm{s}}$-faint objects scattering into the colour-selection region, we

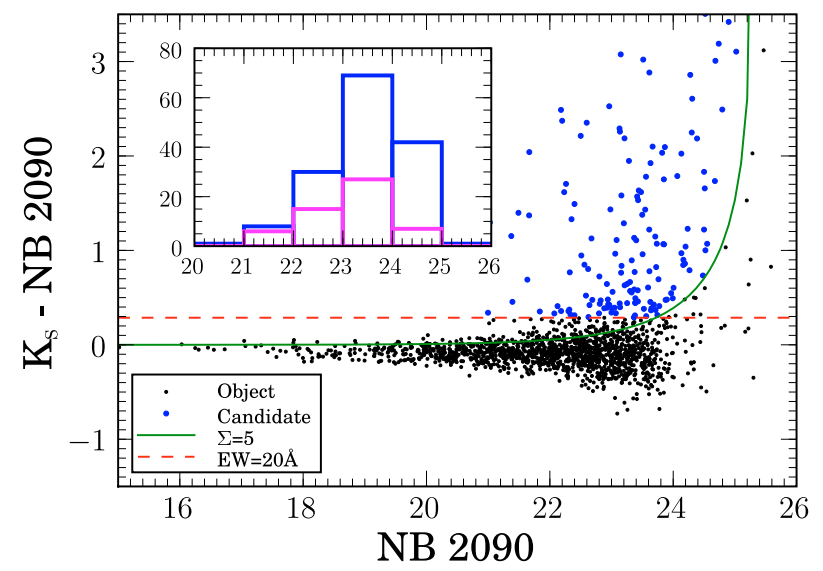

Fig. 1. The selection function for candidate $\mathrm{H} \alpha$ emitters. Black dots represent all the objects detected in the NB2090 image. The red dashed line shows the colour cut of $E W_{\mathrm{H} \alpha}=20 \AA$, and the green solid line the limit of $\Sigma=5$. The blue points show the selected candidate galaxies. The inset histogram shows the narrowband magnitude distribution of all candidate emission line galaxies in blue, and confirmed $z \approx 2.2$ $\mathrm{H} \alpha$ emitters after the rejection of interlopers (see text) in magenta.

required the narrowband flux to be a factor of $\Sigma=5$ greater than the noise in the continuum image. Figure 1 shows the colourmagnitude selection diagram, including the cuts in $E W_{\mathrm{H} \alpha}$ and $\Sigma$.

After inspecting each candidate by eye, we found 152 objects that could potentially be emitters of any emission line between $\operatorname{Pa} \alpha$ at $z \approx 0.11$ and $\operatorname{Ly} \alpha$ at $z \approx 16$. We then crosscorrelated our candidates with the merged $z$ - and $K_{\mathrm{s}}$-selected GOODS-MUSIC catalogue of Santini et al. (2009), finding 143 matches. The objects for which counterparts were not detected all appear to be genuine narrowband-excess objects, but with equivalent width lower limits so high that their stellar continua are too faint to be detected in the ISAAC $K_{\mathrm{s}}$ or ACS $z$-band images. GOODS-MUSIC provided us with spectral energy distributions (SEDs) between the $U$-band and Sptizer-MIPS $24 \mu \mathrm{m}$. To assemble the final catalogue we first examined the spectroscopic redshift measurements in the GOODS-MUSIC catalogue. If the spec- $z$ had a quality flag of "very good" or "good" and was consistent with the redshift interval defined by the NB2090 bandpass $(2.178<z<2.207)$ the galaxy was included (1 galaxy). Using the same quality criterion, we excluded objects if they fell outside that $z$ range (26 galaxies). This ratio of 26 galaxies excluded versus one included may superficially appear to show the selection method in a rather negative light, but the bias can easily be explained by considering the selection methods of the many studies that followed up the GOODS imaging spectroscopically. We searched the spectroscopic catalogues for all galaxies in GOODS-MUSIC with spec- $z$ in the range covered by $F W H M_{N B 2090}$ and spec- $z$ flags of good or better, finding only a single object that could be detected by our survey: the one we do find, followed up from $B z K$ selection. In contrast the catalogue is rife with spectra targeting the $z=0-2$ domain and Lyman-break galaxies at $z>3.5$, and the combined catalogue is heavily biased against $z=2-3$. We find typically as interloping lines: $z \sim 3.2[\mathrm{O} \mathrm{III]}$; low $z \mathrm{~Pa} \alpha, \mathrm{Pa} \beta$ and lines in the higher order Brackett series; and $z \sim 0.28$ [Fe II] emitters, although a handful of more unknown or interesting lines are also recovered. The number of emission line candidates is of sufficient interest to warrant further examination, which will be the topic of a forthcoming paper. For objects with no spec- $z$ or an uncertain flag, we used the Hyper- $z$ photometric-redshift code (Bolzonella et al. 2000), modified to include nebular continuum and lines 
M. Hayes et al.: The H-alpha luminosity function at redshift 2.2
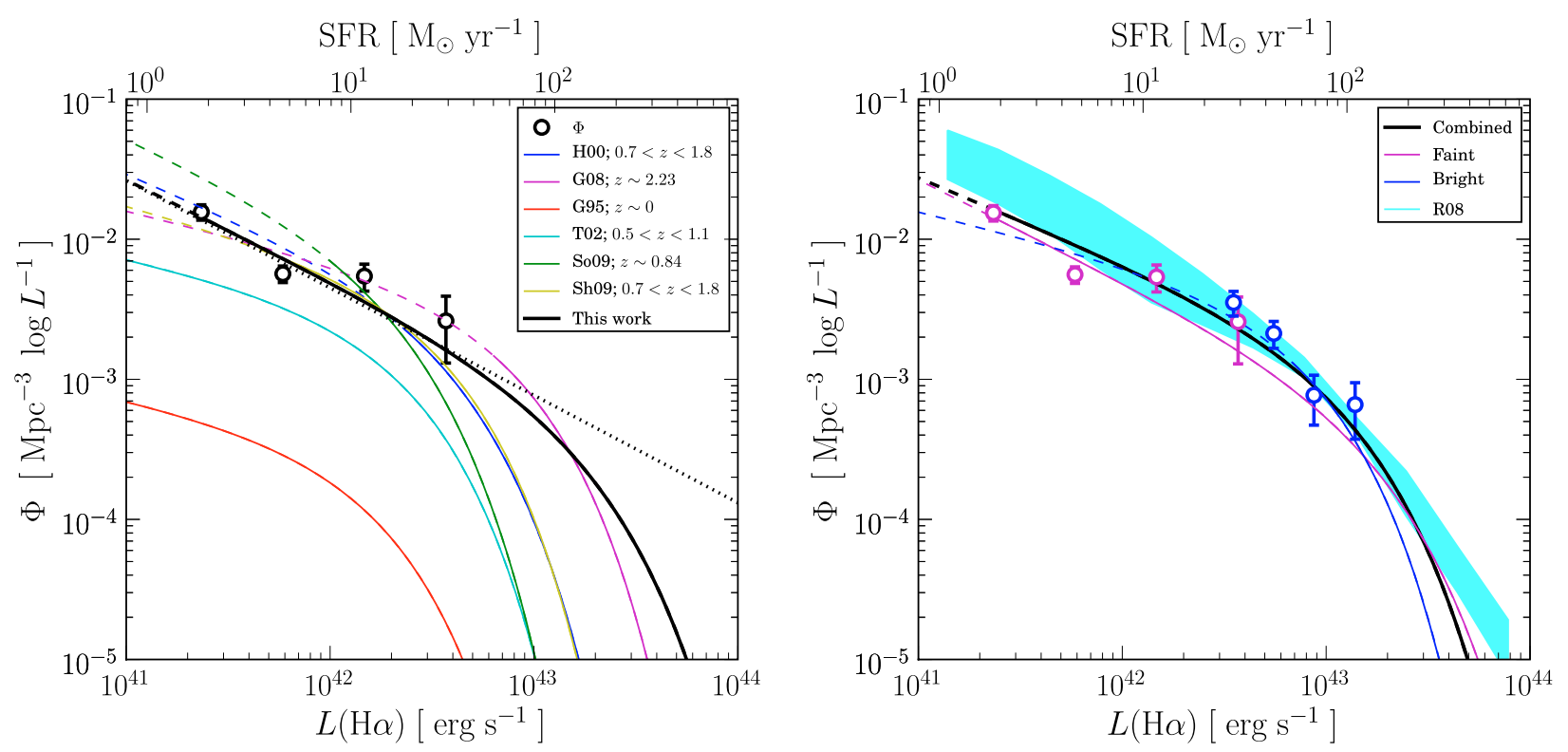

Fig. 2. Left: $\mathrm{H} \alpha$ luminosity functions. The black points show the bins created from HAWK-I data obtained for our programme with error-bars derived from Poisson statistics and Monte Carlo incompleteness simulations. The best-fit Schechter function is shown by the black solid line, while the black dotted line represents the best fitting simple power-law. Other lines show various luminosity functions from the literature, scaled to our cosmology. H00 = (Hopkins et al. 2000, all points), G08 = Geach et al. (2008), G95 = Gallego et al. (1995), T02 = Tresse et al. (2002), So09 $=$ Sobral et al. (2009). Sh09 = Shim et al. (2009). Dashed vs. solid representation shows the $3 \sigma$ limiting survey depth. Right: the complete luminosity function combining these $H A W K-I$ data with the points from G08. The shaded cyan area shows the $L F(\mathrm{H} \alpha)$ derived by Reddy et al. (2008) from the modelling of $\langle z\rangle=2.3$ colour-selected galaxies.

(Schaerer \& de Barros 2009). Here we selected objects that had $1 \sigma$ errors on their phot $z$ consistent with $z=2.19$. Combined with the spectroscopically confirmed objects this gave us $55 z \approx$ $2.2 \mathrm{H} \alpha$-emitters. For security, we tested our galaxies against the $B z K$ colour criterion of $(B-z)-(z-K) \geq-0.2$ (Daddi et al. 2004) to insure that our objects would be classified as star-forming objects at $z \geq 1.4$. Only four of our 55 objects do not satisfy this criterion, but when examining the $B z K$ colours of the entire narrowband-excess sample (143) we found an additional 7 objects that do. We note also that the $B z K$ criteria neglect the fact that the $B$ and $K$ photometry may be contaminated by the Ly $\alpha$ and $\mathrm{H} \alpha$ emission lines, respectively. Since the number of objects that are classified/declassified by $B z K$ colours are (i.) so similar, (ii.) only a small $(\sim 10 \%)$ fraction of our sample, and (iii.) possibly contaminated by lines, we opt to leave our phot- $z$ selected sample unchanged. Finally, we cross-correlated our sample against the 1 Mega-second Chandra Catalogue (Giacconi et al. 2002; Rosati et al. 2002), but no objects in which the $\mathrm{H} \alpha$ production is obviously dominated by an active nucleus were found, including the objects missed by broadband crosscorrelation. Our final catalogue comprises 55 objects.

\section{Results and discussion}

\subsection{The redshift $2.2 \mathrm{H}$-alpha luminosity function}

All $\mathrm{H} \alpha$ photometry is corrected for the contribution of $[\mathrm{N}$ II $] \lambda \lambda 6548,6583 \AA$ lines. In local starbursts the $[\mathrm{N}$ II] $] / \mathrm{H} \alpha$ ratio varies strongly with metallicity $(Z)$ between $\sim 0$ and 0.6 , and $[\mathrm{N} \mathrm{II}] / \mathrm{H} \alpha$ may plausibly be estimated from $L_{\mathrm{H} \alpha}$ through the luminosity-metallicity relationship. However a significant offset in the $L-Z$ relationship is found at $z \sim 2$ (Erb et al. 2006a), and an application of the local relationship at $z \approx 2$ would be insecure. Furthermore, the Erb et al. (2006a) study is based on galaxies an order of magnitude brighter than those found here,

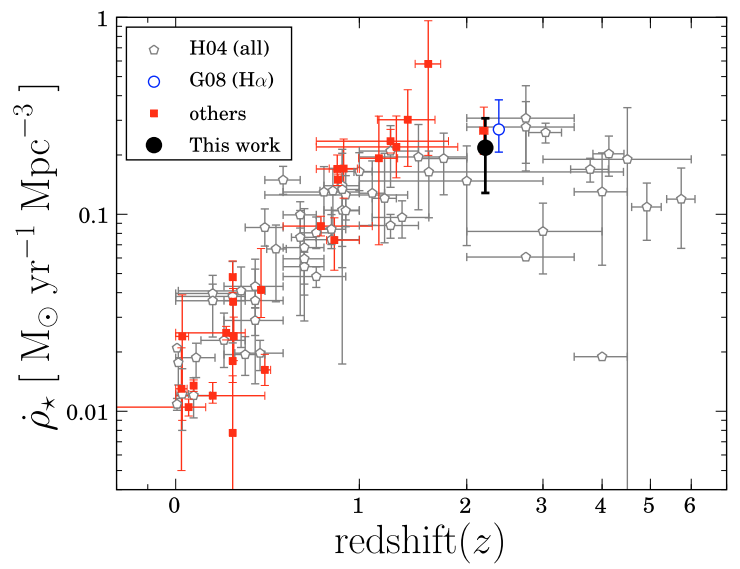

Fig. 3. The cosmic star-formation rate density. Open grey pentagons show all of the dust-corrected points compiled by Hopkins (2004) that were derived from anything other than $\mathrm{H} \alpha$. Filled red squares show dust-corrected points from Shim et al. (2009), all of which are $\mathrm{H} \alpha$-based. The black point is the measurement from this study and the open blue point is the result from G08, artificially shifted to higher- $z$ to allow it to be distinguished from our own.

and we dare not extrapolate to these luminosities. Thus we apply a conservative single correction of $[\mathrm{N} \mathrm{II}] / \mathrm{H} \alpha=0.117$, derived by averaging over the faintest four bins in the data of Erb et al. (2006a).

$L F(\mathrm{H} \alpha)$ is created by binning all selected objects by luminosity, with errors derived from the standard Poisson statistic and Monte Carlo simulations of incompleteness. We fit the Schechter function using a standard $\chi^{2}$ minimiser, determining errors from 1000 re-drawn Monte Carlo realisations. The best-fit Schechter parameters are presented in Table 1 and are shown graphically with other determinations from the literature in Fig. 2. 
Table 1. Luminosity function parameters.

\begin{tabular}{ccccc}
\hline \hline Param. & Unit & HAWK-I all $^{a}$ & HAWK-I $\alpha^{b}$ & Combined $^{c}$ \\
\hline $\log L_{\star}$ & $\mathrm{erg} \mathrm{s}^{-1}$ & $43.22 \pm 0.56$ & $\ldots$ & $43.07 \pm 0.22$ \\
$\log \phi_{\star}$ & $\mathrm{Mpc}^{-3}$ & $-3.96 \pm 0.94$ & $\ldots$ & $-3.45 \pm 0.52$ \\
$\alpha$ & $\ldots$ & $-1.72 \pm 0.20$ & $-1.77 \pm 0.21$ & $-1.60 \pm 0.15$ \\
\hline
\end{tabular}

Notes. ${ }^{(a)} 3$ Schechter parameters: HAWK-I data only. ${ }^{(b)} \alpha$ only: HAWK-I data only. ${ }^{(c)} 3$ Schechter parameters: HAWK-I data + G08 points.

The brightest galaxy in our sample has a luminosity of $3.5 \times 10^{42} \mathrm{erg} \mathrm{s}^{-1}$, which is a factor of 4.5 fainter than the value of $L_{\star}$ we derive, and even allowing for the error on $L_{\star}$, we clearly are not probing this luminosity domain. This galaxy is a factor of 2 fainter than the $L_{\star}=6.7 \times 10^{42} \mathrm{erg} \mathrm{s}^{-1}$ derived by G08 and demonstrates the inadequacy of such a small survey to constrain all Schechter parameters. Indeed, the statistical error on the degenerate parameter $\phi_{\star}$ spans an entire dex and it is apparent that the only parameter we can reliably constrain is $\alpha$, since all our $\mathrm{H} \alpha$ emitters have luminosities that place them firmly in the power-law region of the $L F$. For security, we proceed to examine the faint-end only by fitting of a simple power-law, using exactly the same Monte Carlo realisation data as previously. Our derived faint-end slope is found to be $-1.77 \pm 0.21$, also presented in Table 1, and exhibits a near-identical mean and errorbar.

Some authors find a steepening in the faint end slope of $L F(\mathrm{H} \alpha)$ moving from $z=0$ to $z \gtrsim 1$ with similar results found in UV-selected catalogues at $z=2$ to 3 (Reddy \& Steidel 2009) and $z=6$ (Bouwens et al. 2007). In addition, Hopkins et al. (2000) find a steepening in $\alpha$ for $\mathrm{H} \alpha$ emitters at $z \sim 0.7$ to 1.8 by slitless spectroscopy with the HST/NICMOS, with $\alpha=-1.60$ or 1.86 depending on the selection criteria. However, Trenti \& Stiavelli (2008) note that for such small field-of-view studies, an artificial steepening of the faint-end slope may manifest itself if under-dense regions are targeted. Examining the redshift distribution of galaxies in the whole GOODS field using both spec- $z$ and phot $z$ reveals $z=2.2$ to correspond to neither a significant over- nor under-density. After deriving $\alpha=-1.77 \pm 0.21$, we are able to confirm this increase in the relative abundance of lowerluminosity galaxies at epochs of peak cosmic star-formation. This confirmation comes at the $3 \sigma$ level with respect to Gallego et al. (1995) $(z=0)$, who find $\alpha=-1.35$, and our results are consistent with no evolution within $1 \sigma$ to $z=0.84$ ( $\alpha=-1.65$; Sobral et al. 2009) and $0.8 \lesssim z \lesssim 1.7(\alpha=-1.86$; Hopkins et al. 2000).

\subsection{Combined $L F(H \alpha)$ and the star-formation rate density}

As stated above, our data are not sufficient to constrain the brighter end of $L F(\mathrm{H} \alpha)$. To address this we take the $L F(\mathrm{H} \alpha)$ data-points from G08 and combine them with our own. We extract the $L F$ data-points, which have been corrected for the [N II] using the $z \sim 0$ estimate of Kennicutt \& Kent (1983), and reapply the $z \sim 2$ correction of Erb et al. (2006a) for consistency with our own points. The resulting $L F$ can be seen in the right panel of Fig. 2 with the best-fitting Schechter parameters, derived following exactly the same method as in Sect. 3.1 in Table 1. We also show an estimate of the observed (i.e. dust-uncorrected) $L F(\mathrm{H} \alpha)$ at $\langle z\rangle=2.3$ from Reddy et al. (2008), inferred from colour-selected samples by equating dust-corrected SFR(UV) with $\operatorname{SFR}(\mathrm{H} \alpha)$ and re-applying the effects of dust attenuation to
$\mathrm{H} \alpha$. The observed and inferred $L F(\mathrm{H} \alpha)$ agree well in general, particularly around $L_{\star}$, with only a mild deviation at the faint and (unsampled) bright ends.

In order to estimate the cosmic star-formation rate density, we need to correct our $\mathrm{H} \alpha$ fluxes for dust attenuation. To do this we re-fit the SEDs of all the $\mathrm{H} \alpha$-emitters, fixing their redshift to 2.19. Ideally we would correct our luminosity function bin by bin, but no obvious correlation is observed between $L_{\mathrm{H} \alpha}$ and $A_{V}$. Thus we adopt the average $A_{V}$ for the sample of $1.19 \pm 0.55$, assuming the attenuation law of Calzetti et al. (2000). We do not employ a correction factor for the effects of differential attenuation of stellar and nebular light, since at $z \sim 2$ Erb et al. (2006b) find SFR(UV) equivalent to $\operatorname{SFR}(\mathrm{H} \alpha)$ without this factor. $A_{V}=1.19$ equates to $A_{6563 \AA}=0.977$ for the fainter bins in $L F(\mathrm{H} \alpha)$, identical to the assumption of $1 \mathrm{mag}$ used by G08 at the brighter end, and we correct for 1 mag of attenuation throughout. It is noteworthy that Reddy et al. (2008) find a maximum likelihood $E_{B-V}$ of $0.12\left(A_{6563 \AA}=\right.$ 0.4 , Calzetti dust) for $B X$-selected galaxies at $\langle z\rangle=2.3$. The significantly different extinction determined from $\mathrm{H} \alpha$ selection may be due to the very different selection functions: indeed $E W_{\mathrm{H} \alpha}$ is independent of dust extinction, whereas $B X$ selection cuts the multi-broadband sample based on $U-G$ colours, and thus it is not unreasonable to infer that galaxies with very different extinctions survive the respective cuts. The errors derived on the Schechter parameters are strongly correlated, and thus we compute $\dot{\rho}_{\star}$ from each of the raw Monte Carlo realisations previously described. For consistency with Hopkins (2004) we integrate over the luminosity range $37 \leq \log \left(L_{\mathrm{H} \alpha} / \mathrm{erg} \mathrm{s}^{-1}\right) \leq 47$, where we find a value of $\rho_{\mathrm{L}}=(2.72 \pm 1.14) \times 10^{40} \mathrm{erg} \mathrm{s}^{-1} \mathrm{Mpc}^{-3}$ and $\dot{\rho}_{\star}=(0.215 \pm 0.090) M_{\odot} \mathrm{yr}^{-1} \mathrm{Mpc}^{-3}$. This however represents a significant extrapolation over luminosity, and we also present the same integration performed over the range covered by our bins in total $L F(\mathrm{H} \alpha): 41.5 \leq \log \left(L_{\mathrm{H} \alpha} / \mathrm{erg} \mathrm{s}^{-1}\right) \leq 43.1$. This value, much less dependent on the Schechter parameter fitting, results in $\rho_{\mathrm{L}}=(1.76 \pm 1.02) \times 10^{40} \mathrm{erg} \mathrm{s}^{-1} \mathrm{Mpc}^{-3}$ and $\dot{\rho}_{\star}=(0.139 \pm 0.081) M_{\odot} \mathrm{yr}^{-1} \mathrm{Mpc}^{-3}$. We show the result in Fig. 3, along with two compilations of $\dot{\rho}_{\star}$ from the literature. We include the dust-corrected $\mathrm{H} \alpha$ survey data compiled by Shim et al. (2009), and points from the Hopkins (2004) compilation based upon many different observations where we have excluded all determinations based upon $\mathrm{H} \alpha$. We also indicate separately the point of G08, which has been artificially moved in redshift to separate it from our own. Our point agrees well with that of G08, which is initially surprising, given that we obtain a significantly steeper faint-end slope and marginally higher $L_{\star}$. The culprit behind the similar $\dot{\rho}_{\star}$ yet differing $\left(L_{\star}, \alpha\right)$ is naturally the overall normalisation of the luminosity function, $\phi_{\star}$, which we calculate to be a factor of 4 lower. Furthermore the errorbar produced by our analysis is actually larger than that published by G08, despite our $L F$ extending an order of magnitude fainter in luminosity. These errorbars, however, are statistical errors that result from averaging over many Monte Carlo realisations, and our fitting engine includes an additional parameter $(\alpha)$ - fixing one parameter introduces a systematic error that is not accounted for by Monte Carlo. Had we locked $\alpha$ to its best-fit value and fitted $L_{\star}$ and $\phi_{\star}$ as usual, our statistical errorbar on $\dot{\rho}_{\star}$ would be a factor of 3 smaller. Finally it is possible that our selection criterion of $E W_{\mathrm{H} \alpha}>20 \AA$ could cause us to underestimate the total luminosity density. To assess the impact of this we examine the $E W_{\mathrm{H} \alpha}$ distribution of our sample and fit an exponential of the form $N \propto \exp \left(E W / E W_{0}\right)$, finding $E W_{\mathrm{H} \alpha, 0}=115 \AA$. Assuming the two extremes of $E W_{\mathrm{H} \alpha}$ to be independent of $L_{\mathrm{H} \alpha}$ and also 
of the continuum luminosity, we find that our selection criterion misses between 1.3 and $16 \%$ of the integrated luminosity. We also examine the $E W_{\mathrm{H} \alpha}$ distribution of nearby galaxies in the Sloan Digital Sky Survey, selecting the complete spectroscopic sample at $z<0.1$, and find that removal of galaxies with $E W_{\mathrm{H} \alpha}<20 \AA$ cuts $19 \%$ of the $\mathrm{H} \alpha$ luminosity density.

\section{Summary}

We have used the new $H A W K-I$ instrument mounted at ESO-VLT to carry out a blind, narrowband survey for $\mathrm{H} \alpha$ emitting galaxies at a redshift of 2.2. This is the deepest unbiased survey carried out at this redshift to date and enables us to estimate the faintend of the $\mathrm{H} \alpha$ luminosity function, a parameter hitherto merely assumed at this cosmic epoch. The target is the GOODS-South field, offering us a rich, deep multi-wavelength ancillary dataset, in which we find $55 \mathrm{H} \alpha$ emitters.

1. We construct a luminosity function from the HAWK-I data which we find is well fit by a Schechter function with parameters of $\log L_{\star}=(43.22 \pm 0.56) \mathrm{erg} \mathrm{s}^{-1}, \log \phi_{\star}=$ $(-3.96 \pm 0.94) \mathrm{Mpc}^{-3}, \alpha=(-1.72 \pm 0.20)$. Fitting a single power-law component to all the LF points gives a very similar value of $\alpha=(-1.77 \pm 0.21)$. This confirms the steepening of the faint-end of the galaxy luminosity function out to $z=2$, which is predicted and observed at other wavelengths.

2. We combine our luminosity function bins with those from the much wider but shallower survey of G08, which yields the best-sampled $\mathrm{H} \alpha$ luminosity function at this redshift. This is also well described by a Schechter function with the parameters of $\log L_{\star}=(43.07 \pm 0.22) \mathrm{erg} \mathrm{s}^{-1}, \log \phi_{\star}=$ $(-3.45 \pm 0.52) \mathrm{Mpc}^{-3}, \alpha=(-1.60 \pm 0.15)$.

3. We apply corrections for dust attenuation derived from modelling of the stellar spectral energy distributions and integrate the combined luminosity function to obtain an instantaneous cosmic star-formation rate density of $(0.215 \pm$ 0.090) $M_{\odot} \mathrm{yr}^{-1} \mathrm{Mpc}^{-3}$. This provides the most robust emission-line estimate to date at this redshift.
Acknowledgements. M.H. and D.S. are supported by the Swiss National Science Foundation. G:O. is Royal Swedish academy of Sciences Research Fellow, supported by a grant from the Knut and Alice Wallenberg Foundation and acknowledges support from the Swedish Research Council. We thank Naveen Reddy for making specific $L F(\mathrm{H} \alpha)$ realisations available, Hyunjin Shim for sharing the compilation of literature $\dot{\rho}_{\star}$ values, and Claudia Scarlata for useful discussions. Finally we thank the anonymous referee for thoroughly dissecting the manuscript and suggesting numerous improvements.

\section{References}

Bertin, E., \& Arnouts, S. 1996, A\&AS, 117, 393

Bolzonella, M., Miralles, J.-M., \& Pelló, R. 2000, A\&A, 363, 476

Bouwens, R. J., Illingworth, G. D., Franx, M., \& Ford, H. 2007, ApJ, 670, 928

Bunker, A. J., Warren, S. J., Hewett, P. C., \& Clements, D. L. 1995, MNRAS, 273, 513

Calzetti, D., Armus, L., Bohlin, R. C., et al. 2000, ApJ, 533, 682

Casali, M., Pirard, J.-F., Kissler-Patig, M., et al. 2006, in SPIE Conf. Ser., 6269

Daddi, E., Cimatti, A., Renzini, A., et al. 2004, ApJ, 617, 746

Erb, D. K., Shapley, A. E., Pettini, M., et al. 2006a, ApJ, 644, 813

Erb, D. K., Steidel, C. C., Shapley, A. E., et al. 2006b, ApJ, 646, 107

Gallego, J., Zamorano, J., Aragon-Salamanca, A., \& Rego, M. 1995, ApJ, 455, L1

Geach, J. E., Smail, I., Best, P. N., et al. 2008, MNRAS, 388, 1473

Giacconi, R., Zirm, A., Wang, J., et al. 2002, ApJS, 139, 369

Giavalisco, M., Ferguson, H. C., Koekemoer, A. M., et al. 2004, ApJ, 600, L93

Hopkins, A. M. 2004, ApJ, 615, 209

Hopkins, A. M., Connolly, A. J., \& Szalay, A. S. 2000, AJ, 120, 2843

Kennicutt, Jr., R. C. 1998, ARA\&A, 36, 189

Kennicutt, Jr., R. C., \& Kent, S. M. 1983, AJ, 88, 1094

Lilly, S. J., Le Fevre, O., Hammer, F., \& Crampton, D. 1996, ApJ, 460, L1

Madau, P., Ferguson, H. C., Dickinson, M. E., et al. 1996, MNRAS, 283, 1388

Oke, J. B., \& Gunn, J. E. 1983, ApJ, 266, 713

Pirard, J.-F., Kissler-Patig, M., Moorwood, A., et al. 2004, in SPIE Conf. Ser.

5492, ed. A. F. M. Moorwood, \& M. Iye, 1763

Reddy, N. A., \& Steidel, C. C. 2009, ApJ, 692, 778

Reddy, N. A., Steidel, C. C., Pettini, M., et al. 2008, ApJS, 175, 48

Rosati, P., Tozzi, P., Giacconi, R., et al. 2002, ApJ, 566, 667

Santini, P., Fontana, A., Grazian, A., et al. 2009, A\&A, 504, 751

Schaerer, D., \& de Barros, S. 2009, A\&A, 502, 423

Shim, H., Colbert, J., Teplitz, H., et al. 2009, ApJ, 696, 785

Sobral, D., Best, P. N., Geach, J. E., et al. 2009 [arXiv: 0911.4861]

Trenti, M., \& Stiavelli, M. 2008, ApJ, 676, 767

Tresse, L., Maddox, S. J., Le Fèvre, O., \& Cuby, J.-G. 2002, MNRAS, 337, 369 Yan, L., McCarthy, P. J., Freudling, W., et al. 1999, ApJ, 519, L47 